\title{
Attention to Odor Modulates Thalamocortical Connectivity in the Human Brain
}

\author{
Jane Plailly, ${ }^{1}$ James D. Howard, ${ }^{1}$ Darren R. Gitelman, ${ }^{1,2,3}$ and Jay A. Gottfried ${ }^{1,2,4}$ \\ ${ }^{1}$ Cognitive Neurology and Alzheimer's Disease Center, and Departments of ${ }^{2}$ Neurology and ${ }^{3}$ Radiology, Northwestern University Feinberg School of \\ Medicine, Chicago, Illinois 60611, and ${ }^{4}$ Department of Psychology, Northwestern University Weinberg College of Arts and Sciences, Evanston, Illinois \\ 60208
}

It is widely assumed that the thalamus is functionally irrelevant for the sense of smell. Although animal studies suggest that the mediodorsal (MD) thalamus links primary olfactory (piriform) cortex to olfactory neocortical projection sites in orbitofrontal cortex (OFC), this transthalamic route is regarded to be inconsequential, particularly compared with a direct monosynaptic pathway linking piriform cortex and OFC. In this study, we combined functional magnetic resonance imaging with novel effective connectivity techniques to measure attention-dependent network coherence within direct (nonthalamic) and indirect (transthalamic) olfactory pathways. Human subjects were presented with (or without) an odor and with (or without) a tone, while selectively attending to either modality. Attention to odor significantly modulated neural coupling within the indirect pathway, strengthening MD thalamus-OFC connectivity. Critically, these effects were modality specific (odor $>$ tone attention), directionally sensitive (forward $>$ backward connections), and selective to route (indirect $>$ direct pathway). Our findings support the idea that the human transthalamic pathway is an active modulatory target of olfactory attention. The results imply that olfaction, like all other sensory modalities, requires a thalamic relay, if only to consciously analyze a smell.

Key words: attention; olfaction; mediodorsal thalamus; fMRI; effective connectivity; dynamic causal modeling; human brain

\section{Introduction}

The sense of smell is distinguished from other sensory modalities by its unique anatomical organization (Price, 1990; Carmichael et al., 1994; Shipley and Ennis, 1996). Afferent projections from the nasal periphery remain ipsilateral all the way to primary olfactory (piriform) cortex, and odor inputs can access limbic brain areas in as few as two synapses (Gottfried and Zald, 2005). Moreover, and perhaps most often emphasized in the literature, there is no obligatory thalamic relay between the olfactory receptor layer and olfactory projection sites in neocortex, in contrast to all of the other sensory modalities (Smythies, 1997). Together, these features may help to shape the distinctive functions of human olfaction, including its intimate links to emotion and memory.

Supporting evidence for thalamus-free models of olfaction is essentially based on anatomical work in animal models. These studies suggest that odor information projects from piriform cortex (PC) to olfactory orbitofrontal cortex (OFC) by two different pathways (Tanabe et al., 1975; Yarita et al., 1980; Russchen et al., 1987; Ray and Price, 1992; Carmichael et al., 1994). The principal ("direct") pathway projects directly from PC to OFC and is con-

Received Dec. 18, 2007; revised April 7, 2008; accepted April 10, 2008.

This work was supported by National Institutes of Health/National Institute on Deafness and Other Communication Disorders Grant K08-DC07653 (J.A.G.) and a Fyssen Foundation Fellowship (J.P.). We thank A. P. R. Smith for help on DCM and J.-P. Magué for assistance in programming.

Correspondence should be addressed to Dr. Jay A. Gottfried, Cognitive Neurology and Alzheimer's Disease Center, Northwestern University Feinberg School of Medicine, 320 East Superior Street, Searle 11-453, Chicago, IL 60611. E-mail: j-gottfried@northwestern.edu.

DOI:10.1523/JNEUROSCI.5607-07.2008

Copyright $\odot 2008$ Society for Neuroscience $\quad$ 0270-6474/08/285257-11\$15.00/0 sidered the dominant route for olfactory transmission to neocortical brain areas. The second ("indirect") pathway is a disynaptic projection from $\mathrm{PC}$ to OFC via the mediodorsal (MD) thalamus but is thought to be relatively trivial because of its sparse fiber density (Price, 1985).

The accepted view that olfactory function is independent of a thalamic relay has actually received very little empirical support. Whether the thalamus is pivotal to the sense of smell has important implications for neurobiological models of sensory perception. The thalamus is generally regarded as key for mediating conscious attention toward visual, auditory, somatosensory, and gustatory stimulation (John, 2001; Guillery and Sherman, 2002; Jones, 2006) and is critical for state-dependent gating of sensory information to higher-order neocortical regions (Stériade and Llinàs, 1988; McCormick and Bal, 1994; Jones, 2006). If the thalamus were shown not to be involved in odor perception, then this would help corroborate the idea that the functional neurobiology of the olfactory system is organized profoundly differently from other sensory systems (Murakami et al., 2005; Shepherd, 2005; Kay and Sherman, 2007).

Given that attention is an effective mechanism to induce statedependent changes in sensory gating (Steinmetz et al., 2000), the present study assessed the functional integrity of the human transthalamic odor pathway during attention to smell. Specifically, we combined olfactory functional magnetic resonance imaging (fMRI) with novel effective connectivity techniques [dynamic causal modeling (DCM)] (Friston et al., 2003) to measure attention-dependent network coherence within direct (nontha- 
lamic) and indirect (transthalamic) olfactory pathways. Subjects were presented with (or without) an odor and with (or without) a tone, while selectively attending to either modality. The main prediction was that neural connectivity in the indirect pathway linking PC, MD thalamus, and OFC would be selectively enhanced when subjects attend to odors (vs tones), over and above attention-induced changes in direct (PC to OFC) pathway connections.

\section{Materials and Methods \\ Participants}

Sixteen healthy right-handed volunteers (age range, 22-37 years; mean, 28 years; eight women) without known deficits of smelling, tasting, or hearing provided informed consent to take part in the study, which was approved by the Northwestern University Institutional Review Board. Four subjects were excluded on the basis of technical problems or poor behavioral performance. One subject (J.P.) was an author of this paper.

\section{Stimuli}

Four odorants were selected for their neutral valence and relative unfamiliarity: ( - )rose oxide, butanol, $\alpha$-ionone, and 2-phenylpropionaldehyde (Sigma, St. Louis, MO). Undiluted odorants were absorbed onto diethylphthalate pellets and presented using an MRI-compatible 10-channel computer-controlled olfactometer (airflow set at $2.5 \mathrm{~L} / \mathrm{min}$ ), which permits rapid delivery of odor in the absence of tactile, thermal, or auditory confounds (Gottfried et al., 2002). The auditory tones consisted of four sine-wave sounds of varying frequency: 500, 1000, 1500, and 2000 Hz. The tones were created in Cogent 2000 (http://www.vislab.ucl.ac.uk/ Cogent/), as implemented in Matlab (Mathworks, Natick, MA) and presented to the subject via MR-compatible headphones.

\section{Experimental paradigm}

Subjects were scanned during a selective attention task, in a mixed (block/event) factorial design in which olfactory inputs, auditory inputs, and attentional state were independently manipulated (Fig. 1). This paradigm conformed to a $2 \times 2 \times 2$ design, contrasting olfactory stimulation (odor/no odor), auditory stimulation (tone/no tone), and attentional task (odor attention/tone attention), for a total of eight different conditions. On a given trial, subjects were presented with (or without) an odor and with (or without) a tone. On alternating $60 \mathrm{~s}$ blocks, subjects were instructed to attend to the odor and ignore the tone, indicating whether an odor was present or absent ("odor attention"), or attend to the tone and ignore the odor, indicating whether a tone was present or absent ("tone attention"). Thus, each block of trials involved identical sensory stimulations but differed in terms of attentional processing. Critically, inclusion of the auditory blocks enabled us to control for nonspecific (non-olfactory) effects of attention.

Each block was composed of five trials presented in a pseudorandom order. Each fMRI scan contained four blocks each of odor attention and tone attention. A total of four 10 min scans was obtained, such that each of the eight conditions were presented 20 times over the course of the experiment. An example of one scan is displayed in Figure 1. Subjects were cued to make a $3 \mathrm{~s}$ sniff on viewing a green fixation cross on the monitor of a personal computer that was backprojected onto a mirror mounted in the head coil. During odor attention, odor and no-odor trials were presented for $3.5 \mathrm{~s} \pm 286 \mathrm{~ms}$ (mean $\pm \mathrm{SD}$ ) with a $500 \mathrm{~ms}$ delay, whereas tone and no-tone trials were presented for $2 \mathrm{~s}$ from the green cross appearance. During tone attention, tone and no-tone trials were presented for $2 \mathrm{~s}$ with a $1500 \mathrm{~ms}$ delay, whereas odor and no-odor trials were presented for $2.5 \mathrm{~s} \pm 286 \mathrm{~ms}$ from the green cross appearance. Note that, by including some temporal variability (jitter) in the duration of odor presentation, we were able to minimize state effects related to subject expectancy, which would have had the unwanted effect of reducing attentional load during the detection task. Trials recurred with a mean stimulus-onset asynchrony of $10.2 \mathrm{~s}$ ( \pm variable jitter between 50 and 450 $\mathrm{ms})$. Olfactory, auditory, and visual stimuli presentations were controlled using Cogent 2000. After each trial, subjects pressed one of two buttons to indicate whether an odor or a tone was present or absent (depending on the attentional task). Accuracies of odor detection and

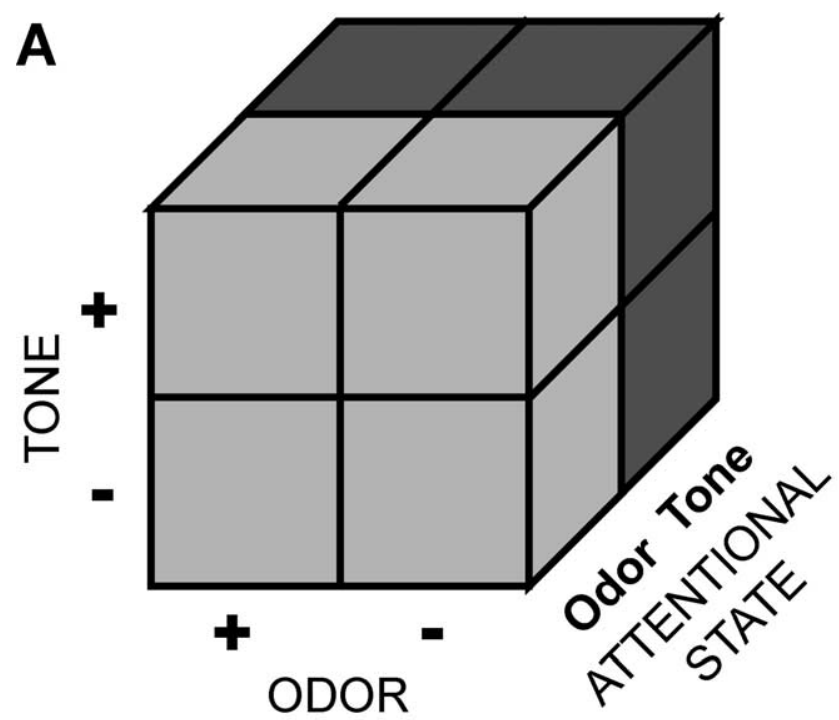

B Block 1

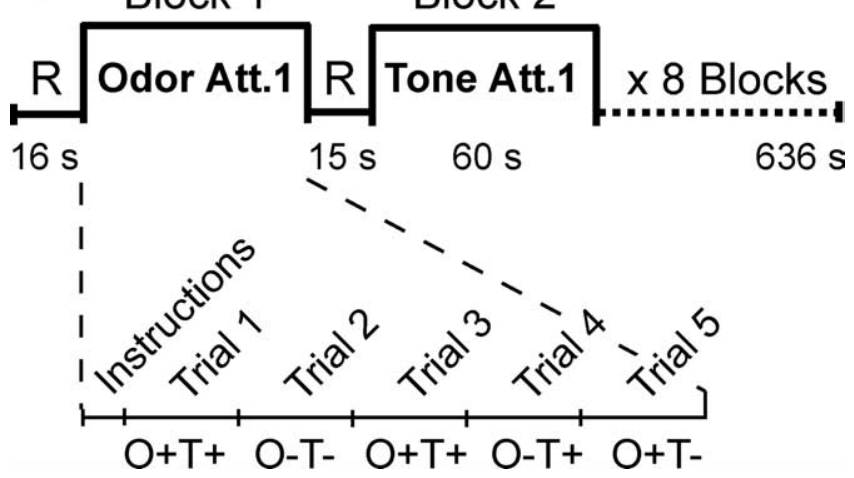

Figure 1. Experimental design. $\boldsymbol{A}$, The study conformed to a fully balanced, three-way factorial design, with factors odor (presence/absence), tone (presence/absence), and attentional task (odor attention/tone attention), resulting in eight different conditions. $\boldsymbol{B}, A$ single fMRI scan (session) was composed of eight 60 s trial blocks alternating between odor attention and tone attention, each separated by a $15 \mathrm{~s}$ rest period $(\mathrm{R})$. Different trial types are specified as follows: odor, tone $(0+\mathrm{T}+)$; odor, no tone $(0+\mathrm{T}-)$; no odor, tone $(0-, \mathrm{T}+)$; no odor, no tone $(0-, T-)$.

tone detection were recorded on-line and averaged across condition types.

\section{Respiratory monitoring}

During scanning, subjects were affixed with a pair of breathing belts to monitor respirations on-line (Gottfried et al., 2002). The output from these belts was transmitted to a piezo-resistive differential pressure sensor, and the resulting analog signal was amplified, digitized, and recorded on a personal computer using a PowerLab 8/30 data acquisition system and accompanying software (ADInstruments, Colorado Springs, CO). Sniff peak amplitude, inspiratory volume, and latency to peak (i.e., sniff duration, from sniff onset time to sniff peak amplitude, marking the end of the inspiratory sniff phase) were computed for each trial. In subsequent analysis, the subject-specific sniff parameters were averaged across each condition and across runs. Each respiratory measure was independently analyzed in Matlab using a separate repeated-measures ANOVA containing three within-subject factors (odor presence/absence, tone presence/absence, and odor/tone attention).

\section{fMRI data acquisition}

Functional imaging was conducted using a Siemens (Munich, Germany) Trio 3T MRI scanner to acquire gradient-echo $\mathrm{T} 2^{*}$-weighted echoplanar images (EPIs) with blood oxygen level-dependent (BOLD) contrast, using an eight-channel head coil and an integrated parallel acqui- 
sition technique known as GRAPPA (GeneRalized Autocalibrating Partially Parallel Acquisition) to improve signal recovery in medial temporal and basal frontal regions ( $\mathrm{Li}$ et al., 2006). Image acquisition was tilted by $30^{\circ}$ from the horizontal axis to further reduce susceptibility artifact in olfactory areas (Deichmann et al., 2003). Four runs of 300 volumes each were collected in an interleaved ascending sequence (24 slices per volume covering PC, OFC, and dorsal thalamus). Imaging parameters were as follows: repetition time (TR), $2 \mathrm{~s}$; echo time, $20 \mathrm{~ms}$; slice thickness, $2 \mathrm{~mm}$; gap, $1 \mathrm{~mm}$; in-plane resolution, $1.72 \times 1.72 \mathrm{~mm}$; field of view, $220 \times 206 \mathrm{~mm}$, matrix size, $128 \times 120$ voxels. Whole-brain high-resolution T1-weighted anatomical scans $\left(1 \mathrm{~mm}^{3}\right)$ were acquired after functional scanning, coregistered to the mean functional image, normalized, and averaged across subjects to aid in localization.

\section{fMRI data preprocessing}

Data preprocessing and analysis were performed using SPM5 (http:// www.fil.ion.ucl.ac.uk/spm/), running under Matlab. After discarding the first six "dummy" volumes of each run to allow for scanner equilibration, the functional images were realigned to the first volume of the first run to correct for subject motion. The images were then spatially normalized to a standard EPI template and smoothed using a three-dimensional Gaussian kernel with full-width half-maximum of $5.16 \times 5.16 \times 9 \mathrm{~mm}$ (three times the voxel size).

\section{fMRI data analysis}

The preprocessed imaging data were modeled using two different methods.

General linear model. In the first approach, the fMRI data were conventionally analyzed in SPM5 using the general linear model (GLM) to estimate the main effects of sensory stimulation and attentional task. Here, each of the eight experimental conditions (varying by odor stimulation, tone stimulation, and task) were modeled independently for each run by convolving condition-specific onset times with a canonical hemodynamic response function (HRF) separately for each subject. This model also included two regressors of no interest (rest and instruction periods), six movement parameters derived from spatial realignment, and three trial-specific sniff parameters (inspiratory volume, peak, and latency/duration) to covary out respiratory effects. The data were highpass filtered (cutoff period of $150 \mathrm{~s}$ ), and temporal autocorrelation was modeled using an $\mathrm{AR}(1)$ process.

For model estimation, condition-specific parameter estimates (pertaining to the height of the HRF) were first obtained via the GLM in a voxelwise manner for each subject. Subsequently, subject-specific linear contrasts of parameter estimates (at the first level) were entered into a three-way factorial design in SPM5 with levels odor stimulation (presence/absence), tone stimulation (presence/absence), and task (odor attention/tone attention), constituting a random-effects analysis. Although this ANOVA generated numerous potential effects and contrasts, we only examined those for which we had a priori hypotheses, including the following: main effects of odor presence and tone presence; main effects of odor attention and tone attention; and interactions of odor presence $X$ attention and of tone presence $X$ attention. For main effects of sensory stimulation and task, significance was set at $p<0.05$ corrected for multiple comparisons, using spheres of $9 \mathrm{~mm}$ radius to define small volumes of interest (VOIs) [small-volume correction (SVC)] (Worsley et al., 1996), based on coordinates from previous human imaging studies of olfactory (Gottfried et al., 2002; Gottfried and Dolan, 2003; Gottfried and Zald, 2005) and auditory (Lockwood et al., 1999; Zatorre and Belin, 2001; Shomstein and Yantis, 2004; Johnson and Zatorre, 2006) processing. [In certain instances, these activations corrected for multiple comparisons across the much larger volume of the whole brain, i.e., whole-brain correction (WBC), signifying responses that were particularly robust.] Activation cluster sizes $(k)$ are also reported (at $p<0.001$ when whole-brain corrected; at $p<0.05$ otherwise). For interaction effects between sensory stimulation and attentional task, we presented activations in a priori regions (including PC, OFC, and MD thalamus) at $p<0.05$ uncorrected. Reported voxels conform to MNI (Montreal Neurological Institute) coordinate space. For presentation, the right side of the images corresponds to the right side of the brain ("neurological" convention).
DCM model. In the second approach, the fMRI data were analyzed in SPM5 using single-state DCM to estimate connectivity strengths (Friston et al., 2003) among brain regions in the direct and indirect (transthalamic) pathways. To optimize the estimation of these parameters and in keeping with the modeling constraints of the DCM technique, data were organized into seven condition types, each concatenated across runs: one "driving input" (all sniff events, regardless of stimuli and task), two "stimulus inputs" (odor and tone), two "modulatory inputs" (odor attention and tone attention), and two conditions of no interest (rest and instruction periods). These onset vectors were encoded either as instantaneous (delta) functions (stimulus conditions) or as $60 \mathrm{~s}$ epochs (task conditions) to assemble seven condition-specific regressors for inclusion in the GLM. We included the three trial-specific respiratory parameters (sniff peak amplitude, volume, and latency/duration) as nuisance regressors, to negate effects of sniffing in the DCM analysis, along with six movement parameters. Model estimation was identical to the GLM model (above) except that it was restricted to single-subject effects. Two $F$ contrasts were computed on a subject-by-subject basis: a general effects-of-interest contrast across the seven conditions, and a more specific contrast testing the effect of the five main experimental conditions (i.e., driving, stimulus, and modulatory inputs only). For additional methodological details, see next section.

\section{Effective connectivity analysis}

DCM has been developed recently to study effective connectivity of the brain (Friston et al., 2003), explicitly for the analysis of functional imaging time series. The term "effective connectivity" refers to the influence that one ensemble of neurons exerts over another compared with "functional connectivity," which is defined as a correlation of activities among remote neurophysiological events (Horwitz et al., 2005). Effective connectivity thus embodies defined models of large-scale neural networks and how differences in stimuli or brain states modulate the interactions between regions in ways that are ecologically meaningful. In DCM, the brain is treated as a "deterministic dynamical" system. It is deterministic because inputs are known and defined, instead of being considered as random. It is dynamic because DCM considers that external inputs perturbate the system and produce changes in neuronal activity, which in turn cause changes in the resulting BOLD signal (output) that is measured with fMRI. Technically, running a DCM analysis involves several steps.

Defining the volumes of interest. Our model incorporated four lefthemisphere regions [anterior PC ( $\mathrm{aPC})$, posterior PC ( $\mathrm{pPC}), \mathrm{MD}$ thalamus, and $\mathrm{OFC}]$ to feature the direct and indirect pathways from aPC to OFC. The separation of PC into aPC and pPC subdivisions was based on previously established anatomical and functional heterogeneity of this structure in both animals and humans (Haberly, 2001; Gottfried et al., 2002; Zelano et al., 2005). The VOIs in aPC, pPC, and MD thalamus were first delineated anatomically, with reference to a human brain atlas (Mai et al., 2004). Because of the large size and extent of the OFC (not all of which is odor responsive), the VOI in OFC was delimited according to the anatomical location of putative olfactory OFC near area 111, as based on a meta-analysis of 26 coordinates from 13 human olfactory imaging studies (Gottfried and Zald, 2005).

In general, a DCM effect for condition A (e.g., odor attention) at regions $\mathrm{Y}$ and $\mathrm{Z}$ (e.g., $\mathrm{MD}$ thalamus and $\mathrm{OFC}$ ) may be observed independently of an overall change in activity for condition $\mathrm{A}$ at regions $\mathrm{Y}$ and $\mathrm{Z}$, highlighting a situation in which DCM can provide information not derived from overall activity. Conversely, to implement DCM, there needs to be a measurable level of neural activity in regions $\mathrm{Y}$ and $\mathrm{Z}$, which is obtained from an $F$ test across all conditions of interest (not just condition A), to ensure that there is sufficient signal variability in the regional time series for estimating the DCM parameters. To this end, we extracted time series of activation for each subject and for each VOI on the basis of $F$ test activation maps representing any combination of effects evoked by all sniff events, odor, tone, odor attention, and tone attention conditions (statistical parametric maps liberally thresholded at $p<0.5$ uncorrected). The VOIs were subsequently defined as 4 -mm-diameter spheres, with centroids of activation within $10 \mathrm{~mm}$ (supplemental Table 
1, available at www.jneurosci.org as supplemental material) of the peak regions observed in an independent odor detection task (see below).

Although we did not have specific predictions about hemispheric differences in network activity, we restricted the DCM analysis to left-sided brain regions, because all subjects showed evidence for significant activity in all olfactory brain regions on the left (but not the right) side, following similar published DCM methods (Smith et al., 2006; Sonty et al., 2007). Moreover, given the strongly ipsilateral nature of olfactory processing, we felt that, as an initial investigation of neural coupling within the direct and indirect pathways, a single-hemisphere model would be reasonably faithful to the general anatomical organization of the olfactory system and would also be simpler to construct without having to make additional assumptions about how to connect regions across hemispheres.

Specifying the intrinsic connections of the model (i.e., how these regions are putatively connected to each other). These connections are typically based on previous anatomical data, but the model does not necessarily reproduce every connection, nor does it imply that connections are monosynaptic. We based the architecture of our model on previous anatomical data in rodents and nonhuman primates (Yarita et al., 1980; Price, 1985; Russchen et al., 1987; Ray and Price, 1992; Carmichael et al., 1994), which demonstrate that PC is intimately connected with olfactory OFC and MD thalamus and that MD thalamus has connections with olfactory OFC. Animal studies further suggest that aPC (being more anteriorly located than $\mathrm{PPC}$ ) receives the majority of afferent inputs from the olfactory bulb and is the major source of direct projections to olfactory OFC (Ray and Price, 1992; Carmichael et al., 1994; Haberly, 2001; Illig, 2005). These studies also indicate that pPC (being more posteriorly located than $\mathrm{aPC}$ ) is the principal source of piriform projections to MD thalamus and that direct and indirect pathways both converge on the same sites in olfactory OFC (Ray and Price, 1992). Given the dense reciprocal nature of these projections, we made no directional assumptions within this model and used bidirectional connections between the brain regions.

Specifying the input connections that drive the model and the modulatory (in this case attentional) connections that modify the neural coupling between regions. In our experiment, subjects made a sniff on each trial, regardless of task. Given that sniffing is known to activate PC, even in the absence of odor (Sobel et al., 1998, 2000), the input or driving effect comprised all sniff events, which drove the model via the aPC. The modulatory connections comprised the two attentional contexts (odor attention and tone attention), the effects of which were tested on each of the intrinsic connections.

Estimating the coupling parameters for each subject. The general procedure consists of a bilinear model to estimate the neurodynamics (i.e., estimated neuronal interactions) and a Balloon-Windkessel model to estimate the hemodynamics (i.e., reproduction of the BOLD data), all using a Bayesian framework to identify the posterior (conditional) distribution of the connectivity parameters, given the data (Friston et al., 2003). In this study, we focused specifically on the changes in effective connectivity induced by the modulatory (attentional) connections, because this was the primary objective of the DCM analysis. By convention (Friston et al., 2003), DCM parameters $>0$ indicate faster or stronger connections from region $\mathrm{A}$ to region $\mathrm{B}$ in the positive direction (i.e., when $\mathrm{A}$ is activated, $\mathrm{B}$ is activated), DCM parameters $<0$ indicate faster or stronger connections from region $\mathrm{A}$ to region $\mathrm{B}$ in the negative direction (i.e., when $A$ is activated, $B$ is deactivated), and DCM parameters $\approx 0$ indicate slower or weaker connections (i.e., when $\mathrm{A}$ is activated, the response in B is poorly coupled, in either direction). Parameter estimates of the intrinsic connections are contained in supplemental Table 2 (available at www.jneurosci.org as supplemental material).

Estimating the contrasts of effective connections at the group level. Subject-specific effective connectivity parameters were entered into a random-effects group analysis to determine significance, in concordance with previous studies (Smith et al., 2006). The modulatory effects of attention on each of the connections was obtained by subtracting the modulatory effect of tone attention from that of odor attention and then testing the significance of these differential effects with one-sample $t$ tests ( $p<0.05$, one-tailed). To the extent that aPC, pPC, MD thalamus, and
OFC are principally linked via excitatory glutamatergic pathways (Tanabe et al., 1975; Yarita et al., 1980; Carmichael et al., 1994; Haberly, 2001), the use of one-tailed $t$ tests enabled us to specifically test the hypothesis that odor (vs tone) attention would strengthen regional coupling in the positive direction (i.e., faster or stronger connections $>0$ ), in keeping with the known basic physiology of these neural circuits. Finally, to compare modulatory effects of attentional context on the direct and indirect pathways, we computed the modulatory effect for the whole indirect pathway by averaging the modulatory effects for the three connections involved in the indirect pathway ( $\mathrm{aPC}$ to $\mathrm{pPC}$, $\mathrm{pPC}$ to $\mathrm{MD}$ thalamus, MD thalamus to OFC) and comparing this with the direct pathway (aPC to OFC).

\section{Independent localizer (odor detection) task}

An independent fMRI run was used to localize odor-responsive cortex and to constrain functional regions of interest in aPC, pPC, OFC, and MD thalamus, as an unbiased method of identifying voxels for inclusion in the DCM analysis. Subjects underwent an odor detection task consisting of 12 odor and 12 no-odor (air only) trials, using four odorants (different from the main study) that were neutral to mildly pleasant in valence, relatively unfamiliar, and matched for intensity (DL-menthol, 2-heptanol, amyl acetate, citronellol; Sigma). Odors (or air only) were delivered using an MRI-compatible olfactometer (airflow, $2.5 \mathrm{~m} / \mathrm{s}$ ). Subjects were cued to make a $2 \mathrm{~s}$ sniff. Odor and no-odor trials were presented for $2 \mathrm{~s}$ in pseudorandom order, with the constraint that equal numbers of condition types appeared in each third of the experiment. Subjects pressed one of two buttons to indicate whether an odor was present or absent. Trials recurred with a stimulus-onset asynchrony of $12 \mathrm{~s}$ ( \pm variable jitter between 75 and $525 \mathrm{~ms}$ ). A total of 160 functional volumes was acquired, using the same imaging parameters as the main experiment. Image preprocessing and analysis were identical to the main experiment, except that temporal and dispersion derivatives were included alongside the canonical HRF. After model estimation in SPM5, we contrasted odor versus no-odor conditions, to generate statistical parametric maps of odor-responsive cortex, on a subject-by-subject basis, liberally thresholded at $p<0.5$ uncorrected.

\section{Results}

\section{Behavioral and physiological data}

Accuracy of odor detection (during odor attention blocks) and accuracy of tone detection (during tone attention blocks) were compared to assess whether task difficulty was matched between the two attentional states. Across the group of subjects, detection accuracy was similar in odor attention $(0.92 \pm 0.017$, mean \pm between-subject SEM) and tone attention $(0.93 \pm 0.016)$, and there was no significant difference in task performance $\left(t_{(11)}=\right.$ 0.63 ; $p>0.53$, two-tailed paired $t$ test), confirming that task demands were kept constant across blocks.

Throughout the experiment, subjects were cued to make a sniff on each trial, regardless of the attentional task, to minimize motor differences between conditions. We subsequently analyzed trial-specific sniff parameters, including peak amplitude, inspiratory volume, and latency to peak (duration), using separate ANOVAs for each of these respiratory measures (Fig. 2, Table 1). During odor stimulation, sniffs were deeper and longer when odor was absent than when odor was present $(p<0.05$ for all three sniff parameters), consistent with our previous findings (Gottfried and Dolan, 2003) showing that subjects make larger sniffs when trying to detect an odor that is not there. Moreover, the interaction between attention task (odor vs tone attention) and odor stimulation (presence vs absence) was significant for both sniff peak $(p=0.004)$ and latency/duration $(p=0.032)$ and was significant for inspiratory volume at trend $(p=0.075)$. Post hoc $t$ tests revealed that subjects sniffed more deeply when odor was absent (vs present) only during odor attention (for the three parameters, $p \leq 0.05$ ) but not during tone attention ( $p>$ 
A Odor Attention Tone Attention
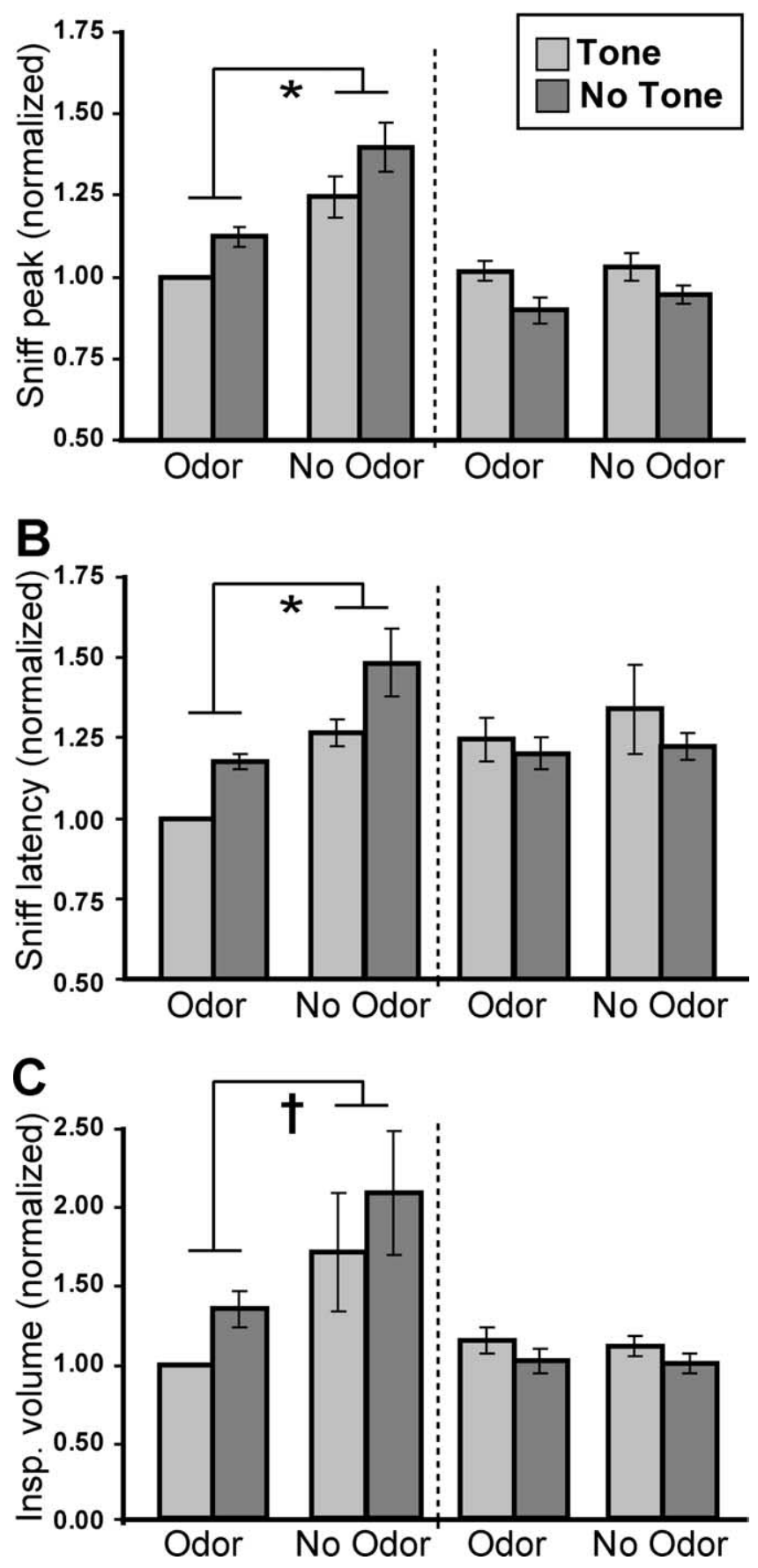

Figure 2. Attentional effects on sniff behavior. $A-C$, Condition-specific respiratory measurements including sniff peak amplitude $(\boldsymbol{A})$, latency to sniff peak $(\boldsymbol{B})$, and inspiratory (insp.) volume $(\boldsymbol{C})$ are shown averaged across subjects (mean \pm between-subject $S E M)$. For display, sniff data were normalized within subjects by setting the first condition (in which both odor and tone were presented during odor attention) to a value of 1.0 and scaling the remaining conditions (Gottfried et al., 2002). These findings show that subjects sniffed more deeply when odor was absent but only during odor attention, highlighting a selective behavioral effect when attention is directed to the olfactory modality. ${ }^{*} p<0.05$; ${ }^{\dagger} p<0.1$.

0.35). These physiological findings highlight the efficacy of our attentional manipulation, whereby subjects attended more to odor content when it was relevant for the task (odor attention) than when it was irrelevant (tone attention). Moreover, the data are compatible with previous work showing that human can se- lectively direct their attention to the olfactory modality (Spence et al., 2001; Zelano et al., 2005).

Imaging data: effects of sensory stimulation and attention We first performed a random-effects fMRI analysis to identify sensory-related and attention-related increases in the magnitude of brain activation (Penny and Ashburner, 2003). Importantly, the fully balanced factorial design of our study enabled us to characterize the main effects of sensory stimulation and attention independently, as well as the interaction between these factors.

The main effect of olfactory stimulation was tested to highlight attention-independent odor-evoked responses in the human brain. This revealed extensive neural activity throughout the key olfactory projection sites (Fig. 3), including bilateral pPC (29, $2,-21: k=162, Z=5.72, p<0.05 \mathrm{WBC}$; and $-17,-5,-21: k=$ $159, Z=4.73, p<0.05 \mathrm{WBC})$, bilateral aPC $(40,9,-18: k=193$, $Z=3.58, p<0.05$ SVC; and $-22,10,-21: k=133, Z=3.14, p=$ 0.06 SVC), and bilateral OFC $(28,28,-12: k=122, Z=4.36, p<$ 0.05 WBC; and $-24,29,-12, k=210, Z=4.11, p<0.05$ SVC). Condition-specific plots and fMRI time courses (Fig. 3) both demonstrate that odor presence (vs absence) evoked greater mean responses in $\mathrm{pPC}$, aPC, and OFC, regardless of whether subjects were asked to attend to odor or attend to tone. These results accord with previous imaging studies (Sobel et al., 2000; Poellinger et al., 2001; Gottfried et al., 2002) and validate the robustness of our paradigm to elicit widespread activation in the olfactory brain.

Notably, and in contrast to previous findings (Sabri et al., 2005; Zelano et al., 2005), none of the above regions was significantly activated when subjects specifically attended to odor. The main effect of odor attention (collapsed across odor and tone) failed to reveal attention-dependent neural activity anywhere in pPC, aPC, or OFC, or MD thalamus, even at a reduced threshold ( $p<0.05$, uncorrected). Interestingly, it was only when we examined the attention $\times$ odor interaction that the effects of attention became evident and only then at liberal thresholds. Medial aspects of olfactory $\mathrm{OFC}(-7,34,-18: k=78, \mathrm{Z}=2.66 ; 19,34$, -9: $k=13, Z=2.00$; and $-17,31,-18: k=15, Z=1.87 ; p$ values $<0.05$ uncorrected) (Fig. 3 ) and areas adjacent to the olfactory tubercle $(-3,12,-18: k=11, Z=2.26, p<0.05$ uncorrected) were sensitive to the presence of odor but only during olfactory (but not auditory) attention. Post hoc $t$ tests confirmed that these interactions were selectively driven by odor stimulation effects during odor attention, in direct comparison with tone attention ( $p$ values $<0.05$ uncorrected). Thus, the OFC and olfactory tubercle responded to the presence of an odor only when it was task relevant (e.g., during odor attention), effectively tuning out olfactory sensory information when it had no bearing on the task (e.g., during tone attention). Although the effect in olfactory tubercle was observed at low threshold, it nevertheless converges with previous fMRI findings from other investigators showing that attention to sniff content modulates activity in this particular brain area (Zelano et al., 2005).

Although auditory-evoked fMRI activity was not a focus of this study, we note that the main effect of tone stimulation (tone presence vs absence) was associated with bilateral increases of fMRI activity in auditory cortices, centered within the anterior aspect of the superior temporal lobe $(67,-21,-3: k=75, Z=$ $3.54, p<0.05$ SVC; and $-48,-33,-3: k=31, Z=2.56, p<0.05$ uncorrected) (Fig. 4). In comparison, the main effect of auditory attention revealed activations in bilateral primary and adjacent auditory cortices spanning the superior temporal gyrus, Heschl's (transverse temporal) gyrus, and the superior temporal sulcus 
Table 1. Summary of statistical results for the behavioral analyses of sniff peak amplitude, sniff latency-to-peak (duration), and sniff inspiratory volume

\begin{tabular}{|c|c|c|c|c|c|c|c|c|c|c|c|c|c|c|}
\hline & \multicolumn{2}{|c|}{ M.E. attention } & \multicolumn{2}{|c|}{ M.E. odor } & \multicolumn{2}{|c|}{$\begin{array}{l}\text { Inter. attention X } \\
\text { odor }\end{array}$} & \multicolumn{2}{|c|}{ M.E. tone } & \multicolumn{2}{|c|}{$\begin{array}{l}\text { Inter. attention } \times \\
\text { tone }\end{array}$} & \multicolumn{2}{|c|}{ Inter. odor $\times$ tone } & \multicolumn{2}{|c|}{$\begin{array}{l}\text { Inter. attention } \\
\times \text { odor } \times \text { tone }\end{array}$} \\
\hline & $F$ & $p$ & $\bar{F}$ & $p$ & $\bar{F}$ & $p$ & $\bar{F}$ & $p$ & $F$ & $p$ & $\bar{F}$ & $p$ & $\bar{F}$ & $p$ \\
\hline Peak & 46.71 & 0.000 & 15.96 & 0.002 & 13.314 & 0.004 & 1.10 & 0.317 & 29.55 & 0.000 & 0.49 & 0.497 & 0.01 & 0.915 \\
\hline Latency & 0.36 & 0.560 & 8.37 & 0.015 & 6.0 & 0.032 & 6.91 & 0.023 & 8.23 & 0.015 & 0.11 & 0.747 & 0.53 & 0.483 \\
\hline Ins. vol. & 4.76 & 0.052 & 5.82 & 0.034 & 3.88 & 0.075 & 9.14 & 0.012 & 14.46 & 0.003 & 0.034 & 0.851 & 0.00 & 0.989 \\
\hline
\end{tabular}

M.E., Main effect; Inter., interaction; Ins. vol., inspiratory volume. For all comparisons, $F$ degrees of freedom $=(1,11)$. Note that a separate ANOVA was conducted for each sniff parameter.

A
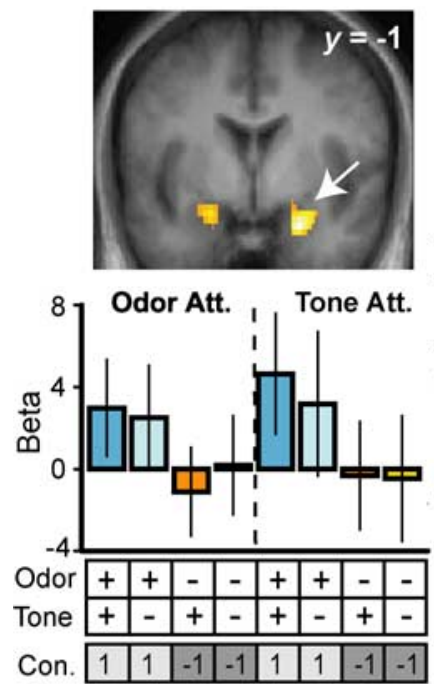

Posterior Piriform

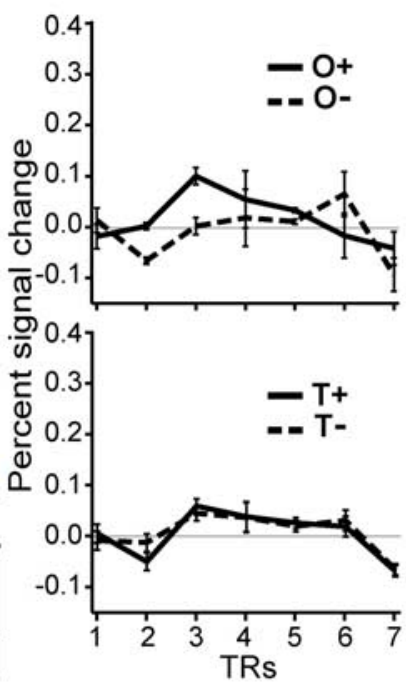

B

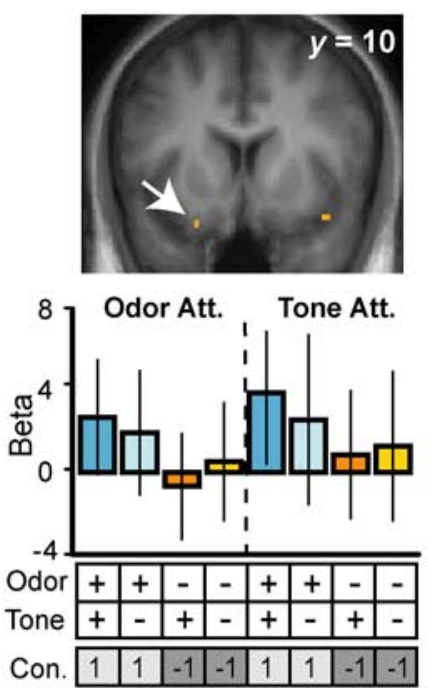

Anterior Piriform

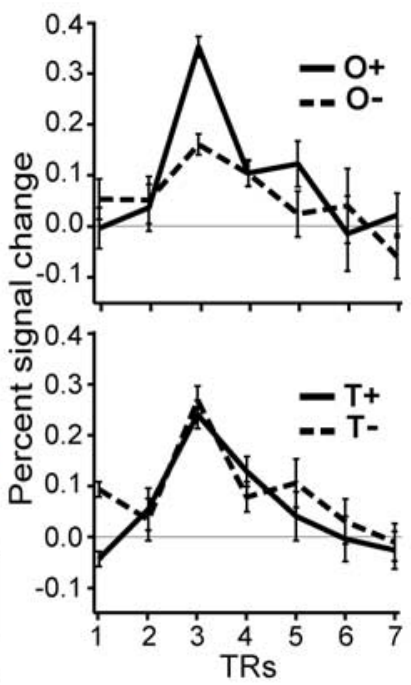

C
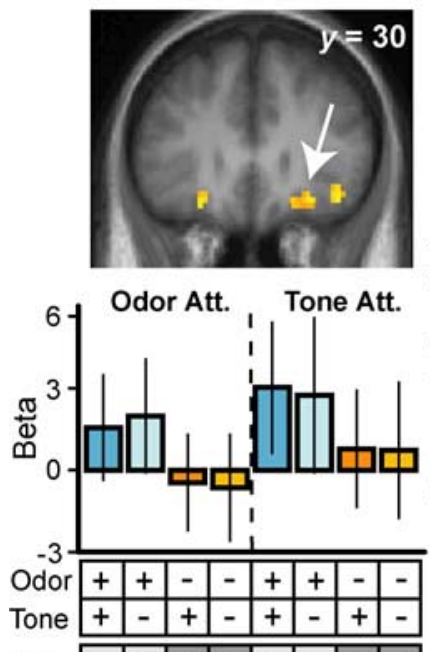

Con. \begin{tabular}{|l|l|l|l|l|l|l|l|}
\hline 1 & 1 & -1 & -1 & 1 & 1 & -1 & -1 \\
\hline
\end{tabular}
OFC

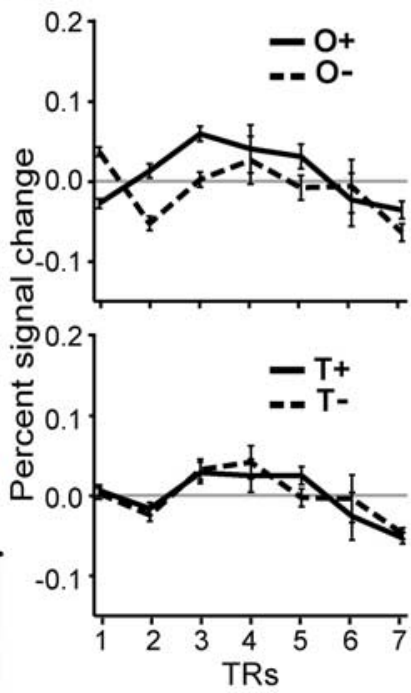

D

OFC

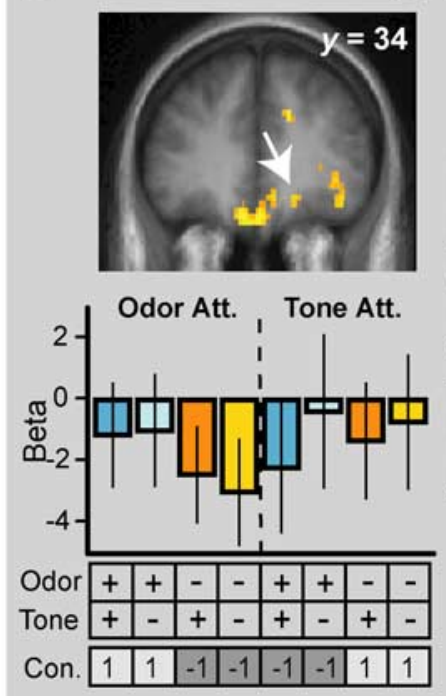

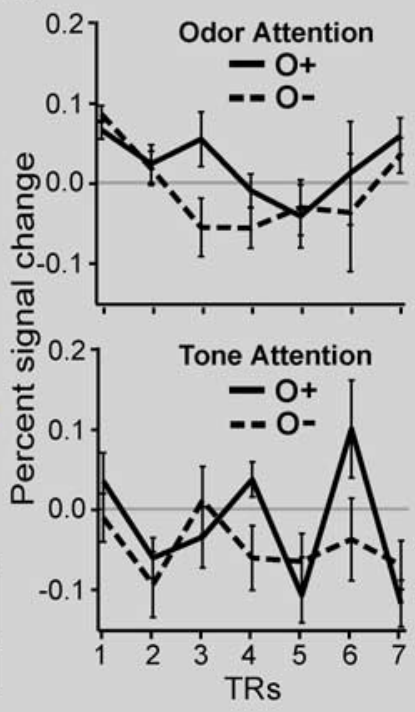

Figure 3. Imaging effects of odor stimulation and olfactory attention. $\boldsymbol{A}-\boldsymbol{C}$, Attention-independent odor-evoked neural activations (main effect of odor) were observed bilaterally in posterior piriform cortex $(\boldsymbol{A})$, anterior piriform cortex $(\boldsymbol{B})$, and olfactory 0FC (C). Neural responses are shown on coronal sections of the mean T1-weighted anatomical scan (threshold for display, $p<0.001$ ). Condition-specific response plots (betas) illustrate greater mean activation when odor was present than when it was absent ( \pm SEM, across-subjects adjusted), regardless of whether subjects attended to odor or tone. To the right of each functional scan, condition-specific response time courses ( \pm SEM, across-subjects adjusted) show that, in each region, neural activity elicited by odor $(0+)$ exceeded that elicited by no odor $(0-)$, with a maximal difference at $2-3$ TRs $(4-6 \mathrm{~s})$, whereas there was no significant difference between tone (T+) and no-tone (T-) trials. The first TR corresponds to the onset of the stimulus, and each TR represents 2 s of scanning. $D$, Although none of these olfactory regions was activated by the main effect of odor attention, there was a significant interaction between odor presence and odor attention in the medial aspect of olfactory 0FC (threshold, $p<0.05$ ), signifying that this brain area responded to odor only when subjects explicitly attended to smell. The effect of olfactory stimulation $(0+$ vs $0-$ ) on the fMRI time courses is evident during odor attention but not during tone attention. Con., Contrast weighting. 
A
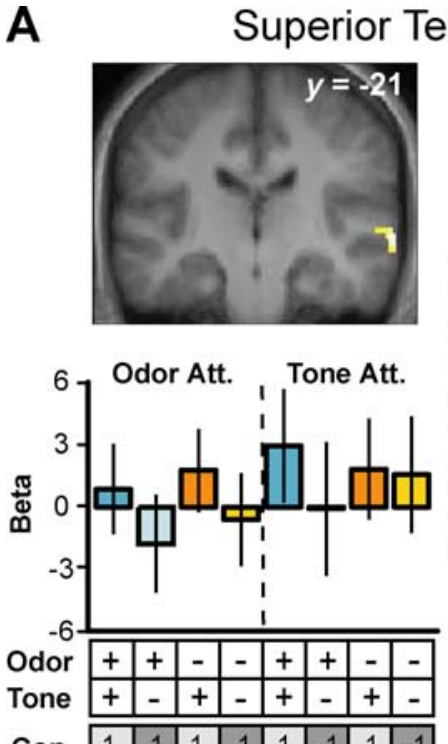

Con.

Odor Att. Tone Att.

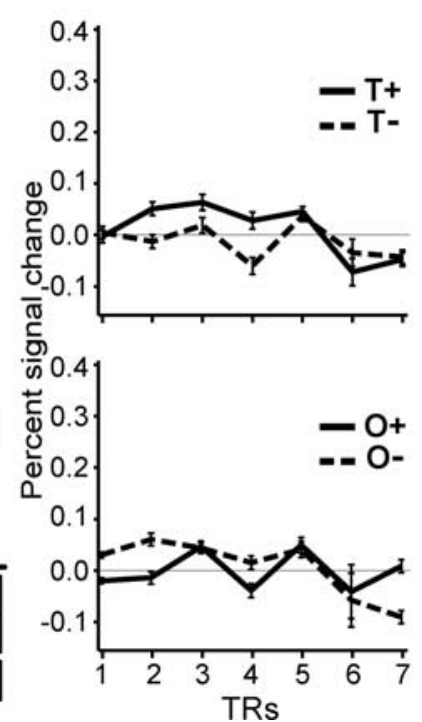

B
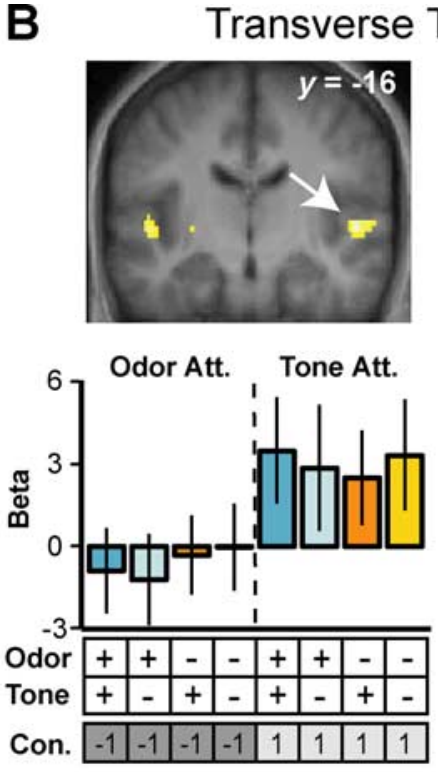

Temporal Gyrus
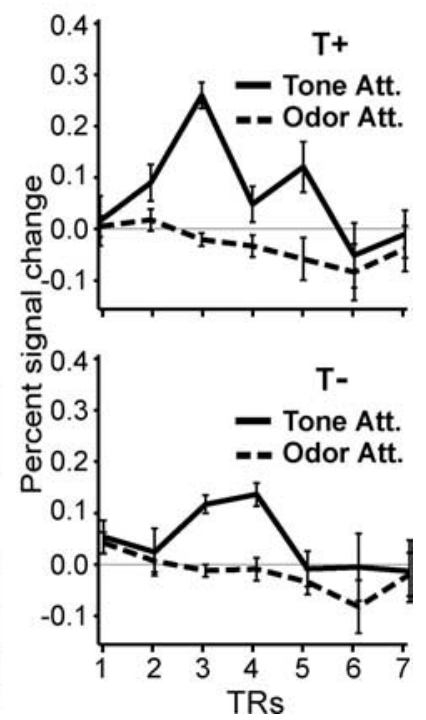

Figure 4. Imaging effects of auditory stimulation and tone attention. $\boldsymbol{A}$, The main effect of tone (presence vs absence) was associated with fMRl activation in auditory cortex, independently of tone attention. Images are thresholded for display at $p<0.01$. Group-averaged fMRI time courses show greater signal change during tone ( $\mathrm{T}+$ ) than no-tone ( $\mathrm{T}-$ ) stimulation, in the absence of an effect of odor stimulation $(0+$ vs $0-$ ). $\boldsymbol{B}$, The main effect of tone attention (Att.) revealed activations in bilateral primary and adjacent auditory cortices, independently of tone stimulation. Corresponding time course profiles from the transverse temporal gyrus show greater responses to tone (vs odor) attention, in both the presence $(T+)$ and absence ( $(T-$ ) of tone.

$(52,-19,0: k=194, Z=3.59, p<0.05$ SVC; $-48,-14,0: k=$ 223, $Z=3.21, p=0.05$ SVC) (Fig. 4), areas previously observed in selective attention to auditory stimuli (Shomstein and Yantis, 2004; Johnson and Zatorre, 2006). In the same regions, neural activity was also seen (at reduced threshold) in the interaction between tone stimulation and tone attention $(58,-15,-3: k=9$, $Z=2.04$; and $-40,-17,0: k=96, Z=3.00 ; p$ values $\leq 0.05$ uncorrected) (data not shown). These additional results further validate the efficacy of our experimental paradigm to induce robust switches between olfactory and auditory attentional modes, with selective effects on sensory-specific cortices.

\section{Imaging data: attentional modulation of olfactory network connectivity}

The central aim of our study was to determine whether attention to odor (vs tone) alters neural connectivity within the putative transthalamic olfactory network. Because odor attention per se did not increase the mean level of activity in MD thalamus (as discussed above), we hypothesized that attentional influences on odor processing might instead be expressed as an increase in thalamocortical coupling within the indirect (transthalamic) odor pathway. Such a mechanism would accord closely with experimental models, suggesting that sensory perception is linked to network synchrony and oscillations throughout thalamocortical circuitry (Jones, 2001; Ribary, 2005).

We used a novel imaging analysis technique known as DCM (Friston et al., 2003) to estimate changes in effective connectivity (Friston et al., 1997) among prespecified olfactory brain regions as a function of attentional context. Based on current anatomical knowledge of olfactory connectivity (see Materials and Methods), we specified a network model of brain regions including aPC, pPC, MD thalamus, and olfactory OFC (Fig. 5). In a subsequent step, the fMRI time series from each of these regions were extracted to test how connection strengths in the direct and indirect pathways were modulated as a function of attention. The

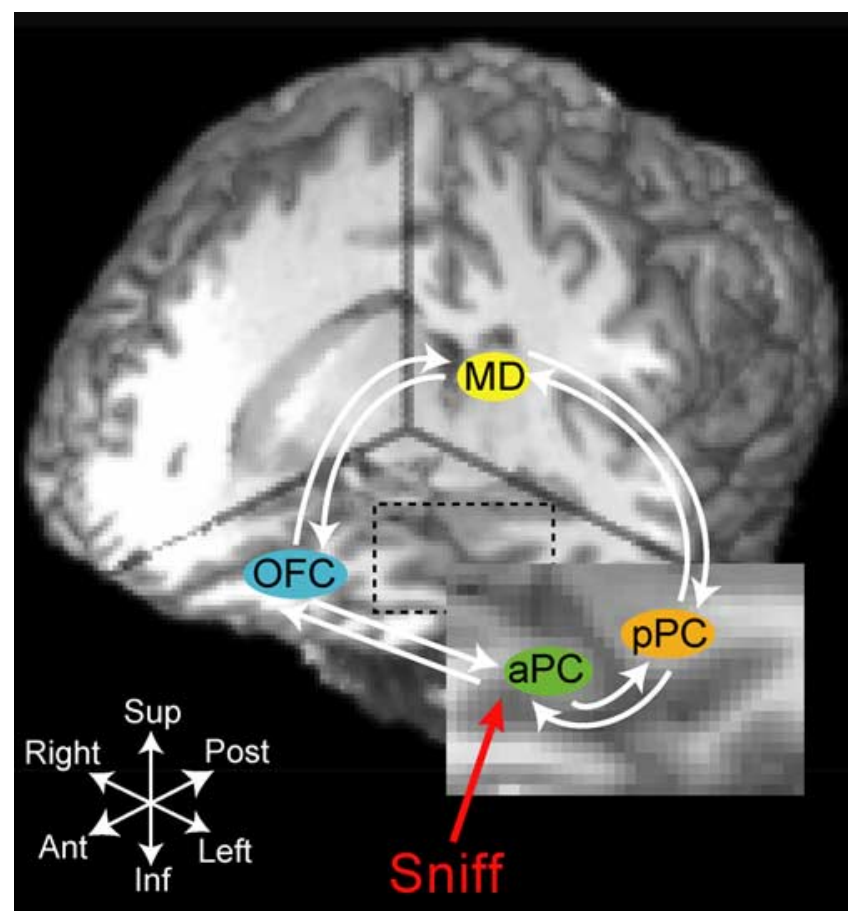

Figure 5. Anatomical schematic of the dynamic causal model specified for testing attentional influences on olfactory network interactions. This model included aPC, pPC, MD thalamus, and $\mathrm{OFC}$, and connections between regions were reciprocal. Sniff events were entered into the network as a direct input to aPC. From aPC, information was disseminated through the network to $\mathrm{OFC}$ along either of two pathways: directly to $\mathrm{OFC}$ (direct pathway) or indirectly via $\mathrm{pPC}$ and MD thalamus (transthalamic pathway). Brain regions are depicted on a rendered threedimensional, T1-weighted brain section, with the left anterosuperior quadrant removed to expose the relevant olfactory areas. The region demarcated within the dashed box is magnified to allow a more precise display of aPC and PPC. Sup, Superior; Post, posterior; Inf, inferior; Ant, anterior. 
Table 2. Parameter estimates of attention-dependent changes in neural connectivity (odor $>$ tone attention) between each pair of linked brain regions in the direct and indirect (transthalamic) pathways

\begin{tabular}{|c|c|c|c|c|c|c|c|c|}
\hline \multirow[b]{2}{*}{ Subject } & \multicolumn{2}{|c|}{ Direct pathway } & \multicolumn{6}{|c|}{ Indirect pathway } \\
\hline & aPC to OFC & OFC to aPC & $\mathrm{aPC}$ to $\mathrm{pPC}$ & $\mathrm{pPC}$ to aPC & $\mathrm{pPC}$ to $\mathrm{MD}$ & $M D$ to $p P C$ & $\mathrm{MD}$ to OFC & $\mathrm{OFC}$ to $\mathrm{MD}$ \\
\hline S01 & -0.176 & -0.167 & 0.054 & -0.004 & 0.03 & -0.01 & 0.197 & 0.119 \\
\hline $\mathrm{SO} 2$ & -0.214 & 0.054 & -0.057 & -0.084 & -0.043 & -0.001 & 0.004 & 0.025 \\
\hline SO3 & 0.065 & 0.1 & -0.024 & 0.094 & 0.008 & 0.057 & 0.075 & -0.077 \\
\hline SO4 & -0.033 & 0.000 & 0.007 & 0.000 & 0.000 & 0.00 & 0.000 & -0.00 \\
\hline SO5 & -0.08 & 0.047 & -0.069 & -0.022 & 0.111 & 0.078 & 0.079 & -0.132 \\
\hline S06 & -0.118 & -0.006 & 0.076 & -0.000 & 0.001 & 0.00 & 0.000 & -0.036 \\
\hline S07 & -0.364 & 0.011 & 0.301 & -0.006 & 0.021 & 0.021 & 0.017 & -0.028 \\
\hline S08 & 0.087 & 0.001 & -0.032 & 0.001 & 0.003 & 0.00 & 0.00 & 0.004 \\
\hline S09 & -0.077 & -0.03 & 0.079 & 0.019 & 0.036 & 0.003 & 0.01 & -0.059 \\
\hline S10 & 0.155 & -0.132 & -0.079 & -0.197 & 0.041 & 0.03 & -0.006 & -0.045 \\
\hline S11 & 0.010 & 0.121 & 0.059 & -0.121 & 0.023 & -0.039 & 0.086 & -0.024 \\
\hline $\mathrm{S} 12$ & -0.058 & 0.008 & 0.047 & 0.009 & 0.02 & -0.08 & 0.009 & 0.014 \\
\hline Mean & -0.067 & 0.001 & 0.030 & -0.026 & $0.021^{*}$ & 0.011 & $0.039^{*}$ & -0.020 \\
\hline SEM & 0.041 & 0.024 & 0.030 & 0.022 & 0.010 & 0.009 & 0.017 & 0.018 \\
\hline
\end{tabular}

advantage of this hypothesis-driven (anatomically based) approach is that it is biologically plausible and results in better model fitting than standard connectivity analyses (Friston et al., 2003).

We began by examining the modulatory effect of odor attention on each of the connections specified in the model. To this end, subject-specific estimates of the coupling parameters linking each pair of nodes (regions) were entered into group-level statistical analyses (Smith et al., 2006), contrasting the effect of odor attention to tone attention (Table 2). This approach identified only two connections that were significantly reinforced when subjects attended to odor (vs tone): the forward connection from MD thalamus to OFC $\left(t_{(11)}=2.02 ; p<0.05\right)$ and the forward connection from pPC to MD thalamus $\left(t_{(11)}=2.25 ; p<0.05\right)$ (Fig. 6). Separate inspection of the odor attention and tone attention data revealed that the MD thalamus to OFC connection was positively strengthened (DCM of $0.033 \pm 0.02$ ) during odor attention and was weakened (DCM of $-0.006 \pm 0.01$ ) during tone attention. The $\mathrm{pPC}$ to MD thalamus connection was strengthened both in the positive direction (DCM of $0.012 \pm 0.02$ ) during odor attention and in the negative direction (DCM of $-0.010 \pm$ 0.02) during tone attention. Thus, for both of these critical connections, there was evidence supporting the direct strengthening of olfactory processing via odor attention. None of the remaining connections, including the backward projections within the transthalamic pathway, as well as the connections in the direct (aPC-OFC) pathway in either direction, was significantly modulated by odor (vs tone) attention (all $p$ values $>0.12$ ).

These findings are compatible with the idea that odor attention strengthens the effective forward connections from $\mathrm{pPC}$ to MD thalamus and from MD thalamus to OFC. We next performed several follow-up analyses to confirm that the influence of odor attention on olfactory connectivity was both directionally selective and pathway specific. First, a direct comparison between forward and backward connections demonstrated that the thalamocortical (forward, MD-OFC) connection was significantly more enhanced that the corticothalamic (backward, OFCMD) connection when subjects attended to odor versus tone (mean difference \pm between-subjects SEM, $0.059 \pm 0.020 ; t_{(11)}=$ $2.94 ; p<0.01)$. Elsewhere in the indirect pathway, there were no significant differences between forward and backward connections linking aPC and $\mathrm{pPC}\left(0.056 \pm 0.032 ; t_{(11)}=1.75 ; p>0.05\right)$ or $\mathrm{pPC}$ and $\mathrm{MD}$ thalamus $\left(0.010 \pm 0.009 ; t_{(11)}=1.05 ; p>0.15\right)$, nor did forward and backward connections linking aPC and OFC
A

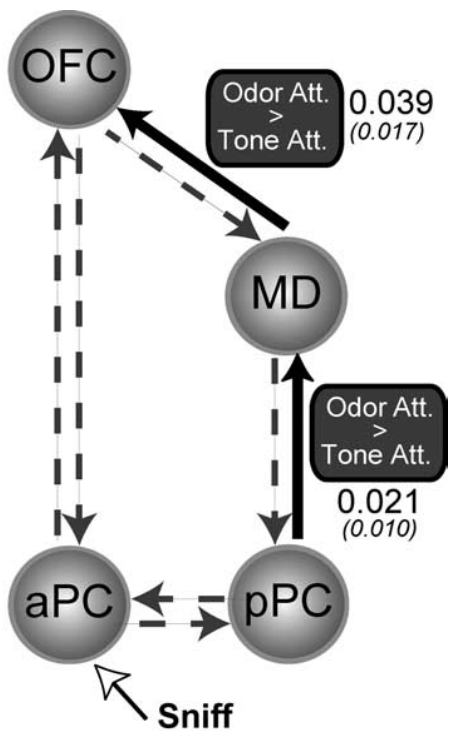

B

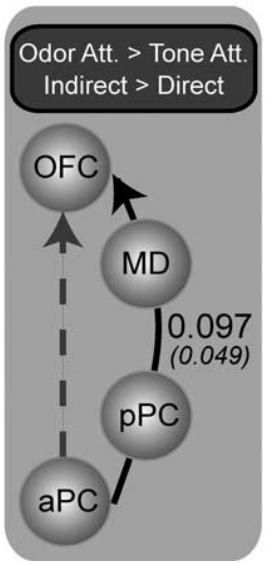

Figure 6. Attention to odor enhances thalamocortical coherence. $\boldsymbol{A}, \boldsymbol{B}, 0 \mathrm{dor}$ attention modulated neural interactions significantly more strongly in the indirect (transthalamic) pathway than in the direct pathway when compared with tone attention. Coupling between pPC and MD thalamus and between MD thalamus and OFC was strengthened (filled black arrows) as a consequence of attending to smell, an effect that was selective for the forward connections $(\boldsymbol{A})$, as well as for the entire indirect pathway $(\boldsymbol{B})$. Group-averaged values for the connectivity parameter estimates (odor - tone attention) are shown beside each of the significant relevant connections [mean (SEM)]. 
significantly differ in the direct pathway $\left(-0.067 \pm 0.047 ; t_{(11)}=\right.$ $-1.42 ; p>0.09$ ).

Second, a comparison between direct and indirect pathways allowed us to test whether odor attention selectively augmented response coupling within the transthalamic network. This analysis showed that, when subjects attended to odors (vs tones), the forward indirect pathway (aPC-pPC-MD thalamus-OFC) exhibited greater modulation than the forward direct pathway (aPC-OFC) $\left(0.097 \pm 0.049 ; t_{(11)}=1.97 ; p<0.05\right)$ (Fig. 6), and the forward indirect pathway was also more modulated than its backward counterpart $\left(0.042 \pm 0.013 ; t_{(11)}=3.19 ; p<0.01\right)$. Finally, the robustness of these effects was corroborated by testing the interaction between pathway and direction, such that connections were significantly strengthened across the indirect (vs direct) pathway for the forward (vs backward) direction during odor (vs tone) attention $\left(0.109 \pm 0.052 ; t_{(11)}=2.08 ; p<\right.$ $0.05)$. It thus appears that feedforward activation across the indirect (transthalamic) pathway is involved in attending to odor.

\section{Discussion}

By combining olfactory fMRI and effective connectivity techniques, we have shown that attention to smell augments functional interactions within thalamocortical networks. Selective attention to odor enhanced the response coupling from $\mathrm{PPC}$ to $\mathrm{MD}$ thalamus and from MD thalamus to olfactory OFC. In addition, odor-specific state-related changes in effective connectivity were enhanced across the entire indirect pathway (from aPC to pPC to MD thalamus to OFC), over and above changes in the direct pathway from aPC to OFC. Together, the findings presented here confirm that the transthalamic pathway is functionally active in humans and is a modulatory target of olfactory attentional processing.

It is important to emphasize that the connectivity analyses were all based on comparisons between odor attention and tone attention, establishing the sensory specificity of our results and ruling out potential confounds related to mere general shifts in arousal or alertness. The possibility of task-related performance differences also seems unlikely, because detection accuracy during odor and tone attention were closely matched (even if we cannot completely rule out the possibility of a ceiling effect), nor can the findings be explained by state differences in sensory features, because odors and tones appeared equal numbers of times during both attentional blocks. Moreover, given that sniffing is known to activate human piriform cortex (Sobel et al., 1998, 2000), trial-specific respiratory parameters were specifically modeled as nuisance covariates in the imaging analyses to regress out the attentive effects on sniffing (compare with Fig. 2; Table 1); that the main effect of odor attention was not associated with significant piriform activity (even at reduced threshold) suggests that any sniff-related confounds were effectively partialed out of the fMRI data. Conversely, because our design did not explicitly test attended versus unattended states, the study was unable to reveal areas that jointly participate in both olfactory and auditory attention, which may account for some of the differences between our findings and those from other studies (Zelano et al., 2005).

The idea that thalamocortical networks assemble conscious perceptual experiences out of each of the senses (except for the sense of smell) continues to motivate research models of attention, cognition, and consciousness. This prevalent view, based primarily on olfactory anatomical evidence from rodents and nonhuman primates, stands in conflict with the strong attentional effect on sniffing behavior observed in our experiment, along with increasing psychophysical data showing that humans can successfully divert their attention between olfactory and nonolfactory modalities, implying a mechanism common to all sensory systems (Spence et al., 2001; Zelano et al., 2005). Our results provide solid neurobiological evidence supporting the functional integrity of an olfactory thalamocortical pathway when a subject attends to odor. The implication is that olfaction, like other sensory modalities, requires a thalamic relay, if only to consciously analyze a smell.

The current data may help to reconcile the disparate findings in the literature regarding olfactory functions of the MD thalamus. In rodents, ablation of this structure impairs olfactory discrimination learning (Eichenbaum et al., 1980; Slotnick and Kaneko, 1981; Staubli et al., 1987; McBride and Slotnick, 1997), particularly for odors that were unfamiliar or difficult to associate (Eichenbaum et al., 1980), and these deficits are overcome only after extensive training (Staubli et al., 1987). In humans, lesions of the medial thalamus have been associated with odor discrimination impairments (Potter and Butters, 1980) as well as defects in the perception of odor valence (Rousseaux et al., 1996). Notwithstanding the functional complexity of MD thalamus, our results suggest a novel way to unify this body of work: a disruption of sensory attention may best explain the olfactory behavioral impairments documented in thalamus-lesioned animals and humans.

Notably, these attention-dependent effects were not accompanied by quantitative changes in thalamic response magnitude (see Results, Imaging data: effects of sensory stimulation and attention), underlining the limitations of conventional fMRI techniques and the potential benefits of effective connectivity approaches. This may actually help explain why MD thalamus is activated only inconsistently in human imaging studies (Sobel et al., 2000; Poellinger et al., 2001; Gottfried et al., 2002), and why increased neural activity in MD thalamus was not observed in two previous imaging studies of odor attention (Sabri et al., 2005; Zelano et al., 2005). Indeed, it is precisely those cases (when "null" results in standard imaging analyses are obtained) that the use of alternative techniques can become uniquely informative. Here the use of dynamic causal modeling enabled us to test mechanistic hypotheses, namely, how the brain implements a particular function rather than simply where the brain implements that function. In the current study, we found that, when subjects attend to an olfactory environment, neural response coupling across regions in the indirect pathway was enhanced, especially connections involving MD thalamus, even if the global activation level of this thalamic area was not.

Based on our pattern of findings, one plausible interpretation is that odor attention induces a state change in the temporal response profile of MD thalamus, or even perhaps in $\mathrm{PPC}(\mathrm{Mu}-$ rakami et al., 2005), thereby facilitating neuronal synchrony throughout the indirect pathway and enhancing effective connectivity as measured with DCM. In contrast, the lack of attention-related increases in mean MD thalamus activity might suggest that there is no net increase in overall spike firing or local processing (Vaadia et al., 1995) at the level of MD thalamus. We believe that such a formulation fits nicely with the known electrophysiological signature of thalamic neurons, which alternate between burst and tonic phases of firing (Jahnsen and Llinàs, 1984) to promote state-dependent alterations in sleep and wakefulness (Stériade and Llinàs, 1988; Llinàs and Pare, 1991) and in consolidating perceptual experience, perhaps orchestrated via synchronized oscillatory activity in the gamma frequency band throughout thalamocortical networks (Llinàs and Ribary, 1993). Of course, the slow temporal resolution of the fMRI technique 
(typically 4-6 s) is not sufficient to discern fast (milliseconds) shifts in temporal coding at the neuronal level, but our data are nevertheless consistent with the idea that attending to smells may have an impact on response timing, rather than response magnitude, within the olfactory transthalamic pathway.

How might the direct and indirect olfactory pathways actually interact during an encounter with an odor stimulus? We speculate that the monosynaptic projection directly linking aPC to olfactory OFC would serve as a quick sweep of the odor environment, permitting fast, more reflexive sensory processing, at a coarse level of discrimination, and perhaps not contingent on conscious awareness. In turn, the transthalamic pathway, with at least two additional intervening synapses between aPC and OFC, would function at a slower timescale, but with a discriminative capacity much more finely tuned, and informed by cognitive factors, such as attention, expectation, and memory (Wilson and Stevenson, 2003). Such a system could serve as an effective gate for the direct pathway, helping to select only those inputs with behavioral relevance for additional processing downstream of OFC. Through feedback connections from OFC, the transthalamic network could even help to refine the receptive field properties in olfactory bulb and aPC, optimizing discrimination for biologically salient odor inputs. Additional work will be necessary to elucidate how exactly these dual pathways cooperate, but our present findings make it clear that the MD thalamus should be incorporated into future research models of olfactory cognitive processing.

\section{References}

Carmichael ST, Clugnet MC, Price JL (1994) Central olfactory connections in the macaque monkey. J Comp Neurol 346:403-434.

Deichmann R, Gottfried JA, Hutton C, Turner R (2003) Optimized EPI for fMRI studies of the orbitofrontal cortex. NeuroImage 19:430-441.

Eichenbaum H, Shedlack KJ, Eckmann KW (1980) Thalamocortical mechanisms in odor-guided behavior. I. Effects of lesions of the mediodorsal thalamic nucleus and frontal cortex on olfactory discrimination in the rat. Brain Behav Evol 17:255-275.

Friston KJ, Buechel C, Fink GR, Morris J, Rolls E, Dolan RJ (1997) Psychophysiological and modulatory interactions in neuroimaging. NeuroImage 6:218-229.

Friston KJ, Harrison L, Penny W (2003) Dynamic causal modelling. NeuroImage 19:1273-1302.

Gottfried JA, Dolan RJ (2003) The nose smells what the eye sees: crossmodal visual facilitation of human olfactory perception. Neuron 39:375-386.

Gottfried JA, Zald DH (2005) On the scent of human olfactory orbitofrontal cortex: meta-analysis and comparison to non-human primates. Brain Res Brain Res Rev 50:287-304.

Gottfried JA, Deichmann R, Winston JS, Dolan RJ (2002) Functional heterogeneity in human olfactory cortex: an event-related functional magnetic resonance imaging study. J Neurosci 22:10819-10828.

Guillery RW, Sherman SM (2002) Thalamic relay functions and their role in corticocortical communication: generalizations from the visual system. Neuron 33:163-175.

Haberly LB (2001) Parallel-distributed processing in olfactory cortex: new insights from morphological and physiological analysis of neuronal circuitry. Chem Senses 26:551-576.

Horwitz B, Warner B, Fitzer J, Tagamets MA, Husain FT, Long TW (2005) Investigating the neural basis for functional and effective connectivity. Application to fMRI. Philos Trans R Soc Lond B Biol Sci 360:1093-1108.

Illig KR (2005) Projections from orbitofrontal cortex to anterior piriform cortex in the rat suggest a role in olfactory information processing. J Comp Neurol 488:224-231.

Jahnsen $\mathrm{H}$, Llinàs $\mathrm{R}$ (1984) Electrophysiological properties of guinea-pig thalamic neurones: an in vitro study. J Physiol (Lond) 349:205-226.

John ER (2001) A field theory of consciousness. Conscious Cogn 10:184-213.

Johnson JA, Zatorre RJ (2006) Neural substrates for dividing and focusing attention between simultaneous auditory and visual events. NeuroImage 31:1673-1681.

Jones EG (2001) The thalamic matrix and thalamocortical synchrony. Trends Neurosci 24:595-601.

Jones EG (2006) The thalamus, Ed 2. Cambridge, UK: Cambridge UP.

Kay LM, Sherman SM (2007) An argument for an olfactory thalamus. Trends Neurosci 30:47-53.

Li W, Luxenberg E, Parrish T, Gottfried JA (2006) Learning to smell the roses: experience-dependent neural plasticity in human piriform and orbitofrontal cortices. Neuron 52:1097-1108.

Llinàs R, Ribary U (1993) Coherent 40-Hz oscillation characterizes dream state in humans. Proc Natl Acad Sci USA 90:2078-2081.

Llinàs RR, Pare D (1991) Of dreaming and wakefulness. Neuroscience 44:521-535.

Lockwood AH, Salvi RJ, Coad ML, Arnold SA, Wack DS, Murphy BW, Burkard RF (1999) The functional anatomy of the normal human auditory system: responses to 0.5 and $4.0 \mathrm{kHz}$ tones at varied intensities. Cereb Cortex 9:65-76.

Mai JK, Assheuer J, Paxinos G (2004) Atlas of the human brain, Ed 2. San Diego: Elsevier Academic.

McBride SA, Slotnick B (1997) The olfactory thalamocortical system and odor reversal learning examined using an asymmetrical lesion paradigm in rats. Behav Neurosci 111:1273-1284.

McCormick DA, Bal T (1994) Sensory gating mechanisms of the thalamus. Curr Opin Neurobiol 4:550-556.

Murakami M, Kashiwadani H, Kirino Y, Mori K (2005) State-dependent sensory gating in olfactory cortex. Neuron 46:285-296.

Penny WD, Ashburner J (2003) Random effects analysis. In: Human brain function, Ed 2 (Frackowiak RSJ, Friston KJ, Frith C, Dolan R, Price C, Ashburner J, Penny W, Zeki S, eds), pp 843-850. San Diego: Academic.

Poellinger A, Thomas R, Lio P, Lee A, Makris N, Rosen BR, Kwong KK (2001) Activation and habituation in olfaction: an fMRI study. NeuroImage 13:547-560.

Potter H, Butters N (1980) An assessment of olfactory deficits in patients with damage to prefrontal cortex. Neuropsychologia 18:621-628.

Price JL (1985) Beyond the primary olfactory cortex: olfactory-related areas in the neocortex, thalamus and hypothalamus. Chem Senses 10:239-258.

Price JL (1990) Olfactory system. In: The human nervous system (Paxinos G, ed), pp 979-1001. San Diego: Academic.

Ray JP, Price JL (1992) The organization of the thalamocortical connections of the mediodorsal thalamic nucleus in the rat, related to the ventral forebrain-prefrontal cortex topography. J Comp Neurol 323:167-197.

Ribary U (2005) Dynamics of thalamo-cortical network oscillations and human perception. Prog Brain Res 150:127-142.

Rousseaux M, Muller P, Gahide I, Mottin Y, Romon M (1996) Disorders of smell, taste, and food intake in a patient with a dorsomedial thalamic infarct. Stroke 27:2328-2330.

Russchen FT, Amaral DG, Price JL (1987) The afferent input to the magnocellular division of the mediodorsal thalamic nucleus in the monkey, Macaca fascicularis. J Comp Neurol 256:175-210.

Sabri M, Radnovich AJ, Li TQ, Kareken DA (2005) Neural correlates of olfactory change detection. NeuroImage 25:969-974.

Shepherd GM (2005) Perception without a thalamus: how does olfaction do it? Neuron 46:166-168.

Shipley MT, Ennis M (1996) Functional organization of olfactory system. J Neurobiol 30:123-176.

Shomstein S, Yantis S (2004) Control of attention shifts between vision and audition in human cortex. J Neurosci 24:10702-10706.

Slotnick BM, Kaneko N (1981) Role of mediodorsal thalamic nucleus in olfactory discrimination learning in rats. Science 214:91-92.

Smith AP, Stephan KE, Rugg MD, Dolan RJ (2006) Task and content modulate amygdala-hippocampal connectivity in emotional retrieval. Neuron 49:631-638.

Smythies J (1997) The functional neuroanatomy of awareness: with a focus on the role of various anatomical systems in the control of intermodal attention. Conscious Cogn 6:455-481.

Sobel N, Prabhakaran V, Desmond JE, Glover GH, Goode RL, Sullivan EV, Gabrieli JD (1998) Sniffing and smelling: separate subsystems in the human olfactory cortex. Nature 392:282-286.

Sobel N, Prabhakaran V, Zhao Z, Desmond JE, Glover GH, Sullivan EV, Gabrieli JD (2000) Time course of odorant-induced activation in the human primary olfactory cortex. J Neurophysiol 83:537-551. 
Sonty SP, Mesulam MM, Weintraub S, Johnson NA, Parrish TB, Gitelman DR (2007) Altered effective connectivity within the language network in primary progressive aphasia. J Neurosci 27:1334-1345.

Spence C, McGlone FP, Kettenmann B, Kobal G (2001) Attention to olfaction. A psychophysical investigation. Exp Brain Res 138:432-437.

Staubli U, Schottler F, Nejat-Bina D (1987) Role of dorsomedial thalamic nucleus and piriform cortex in processing olfactory information. Behav Brain Res 25:117-129.

Steinmetz PN, Roy A, Fitzgerald PJ, Hsiao SS, Johnson KO, Niebur E (2000) Attention modulates synchronized neuronal firing in primate somatosensory cortex. Nature 404:187-190.

Stériade M, Llinàs RR (1988) The functional states of the thalamus and the associated neuronal interplay. Physiol Rev 68:649-742.

Tanabe T, Yarita H, Ino M, Ooshima Y, Takagi SF (1975) An olfactory projection area in orbitofrontal cortex of the monkey. J Neurophysiol 38:1269-1283.
Vaadia E, Haalman I, Abeles M, Bergman H, Prut Y, Slovin H, Aertsen A (1995) Dynamics of neuronal interactions in monkey cortex in relation to behavioural events. Nature 373:515-518.

Wilson DA, Stevenson RJ (2003) The fundamental role of memory in olfactory perception. Trends Neurosci 26:243-247.

Worsley KJ, Marrett S, Neelin P, Vandal AC, Friston KJ, Evans AC (1996) A unified statistical approach for determining significant signals in images of cerebral activation. Hum Brain Mapp 4:58-73.

Yarita H, Iino M, Tanabe T, Kogure S, Takagi SF (1980) A transthalamic olfactory pathway to orbitofrontal cortex in the monkey. J Neurophysiol 43:69-85.

Zatorre RJ, Belin P (2001) Spectral and temporal processing in human auditory cortex. Cereb Cortex 11:946-953.

Zelano C, Bensafi M, Porter J, Mainland J, Johnson B, Bremner E, Telles C, Khan R, Sobel N (2005) Attentional modulation in human primary olfactory cortex. Nat Neurosci 8:114-120. 